\title{
Relative Bioavailability of Zinc in Yogurt Using Body Weight Gain, Femur Weight and Bone Zinc Content in Rats as Markers
}

\author{
FC Tesán ${ }^{1, *}, \mathrm{~N} \mathrm{Collia}^{2}, \mathrm{~S}$ Arnoldi ${ }^{2}, \mathrm{~J} \mathrm{Fuda}^{1}, \mathrm{H} \mathrm{Torti}^{1}$, R Weill ${ }^{3}$, MJ Salgueiro ${ }^{1,2}$ and J Boccio ${ }^{1}$ \\ ${ }^{1}$ Stable Isotope Laboratory Applied to Biology and Medicine; ${ }^{2}$ Radioisotope Laboratory, Physics Department, School of \\ Pharmacy and Biochemistry, University of Buenos Aires, Buenos Aires, Argentina; and ${ }^{3}$ Research and Development \\ Department, Danone Argentina SA, Buenos Aires, Argentina
}

\begin{abstract}
Objective: some previous works have shown the importance of zinc in nutrition, and even evaluated different zinc sources for food supplementation with this micronutrient. The aim of this work was to evaluate zinc gluconate stabilized with glycine (BZ) as a zinc source for food fortification by employing yogurt as the vehicle and zinc sulfate (SZ) as the standard zinc source. Materials and methods: weaned Sprague Dawley rats were separated in 7 groups of 10 rats each, which were fed with diets according to the scheme: Group 1- 2ppm Zn; Groups 2, 3 and 4 - 9, 20 and 34 ppm Zn as $\mathrm{SO}_{4} \mathrm{Zn} .7 \mathrm{H}_{2} \mathrm{O}$ (SZ) and Groups 5, 6 and 7 - 9, 20 and $34 \mathrm{ppm} \mathrm{Zn}$ as zinc gluconate stabilized with glycine (BZ) during 21 days. Zinc sources were provided in a vehicle as yogurt, added in the final diet at $10 \%$. Body weight gain, femur weight and femur zinc content were evaluated at the end of the treatments in order to determine zinc bioavailability, as previously described by others. In this way, body weight gain and femur weight were analyzed by a non linear regression according to equation: $\mathrm{Y}=\mathrm{Ymax}\left(1-\mathrm{e}^{(-\mathrm{kX})}\right)$ and femur zinc content was analyzed by a linear regression, all of them as a function of dietary zinc from each source. Results: relative zinc bioavailability of BZ was $0.95 ; 0.95$ and 1.10 according to body weight gain, femur weight and femur zinc content, respectively. Conclusion: zinc gluconate stabilized with glycine may be considered as a suitable source of zinc for food fortification in a yogurt matrix.
\end{abstract}

Key Words: Zinc, bioavailability, food fortification.

\section{INTRODUCTION}

Zinc deficiency has devastating effects on growth, immune response, cognitive functions, bone and gonadal development [1-3]. The main cause of this deficiency is nutritional, but it can also appear as a consequence of metabolism abnormalities. Therefore, in spite of being a micronutrient, zinc is an essential element [4] that should be provided with the diet according to nutritional requirements $[5,6]$, and if not possible, some strategies such as food fortification or supplementation should be considered. This is a very important matter since the consequences of zinc deficiency on growth can be reverted by restoring normal zinc levels in the diet [7]. Thus, it is of our interest, as well as other researchers, to evaluate the most suitable zinc source for that mentioned strategies to overcome the zinc deficiency problem.

With that intention, the aim of this work was to determine the relative bioavailability of zinc gluconate stabilized with glycine using zinc sulfate as the standard zinc source in a yogurt as the nutritional matrix of the vehicle.

Working with zinc involves one disadvantage: there are no sensitive and reliable markers that can indicate accurately and precisely the nutritional effect of this micronutrient on the host. Therefore, for this experiment we chose to analyze growth parameters such as body weight gain (as an indicator

*Address correspondence to this author at the Radioisotope laboratory, UBA. Junin 956 piso bajo, 1113, Ciudad de Buenos Aires, Argentina; Tel/Fax: (+5411) 4964-8202; ext: 33; E-mail: ftesan@ffyb.uba.ar of total body growth) and femur weight (as the same for bone growth) as well as femur zinc content as markers for zinc bioavailability.

\section{MATERIALS AND METHODS}

\section{Animals and Diets}

The experiment was performed according to the Guide for the Care and Use of Laboratory Animals, U.S. National Research Council, 1996.

We used 70 female Sprague Dawley weaned rats (School of Pharmacy and Biochemistry, University of Buenos Aires, Argentina), that were kept in $12 \mathrm{~h}$ light followed by $12 \mathrm{~h}$ darkness cycles during the experiment, and housed in stainless steel cages with grated floor and collection trays.

The rats were randomly separated so as to form 7 groups of 10 rats each, which were fed with different diets, all based on the recommendations of the AIN 93-G but without the addition of zinc as described elsewhere [8,9]. Instead, zinc was provided at different levels in the same vehicle as yogurt, from two different sources to be evaluated as zinc sulfate (SZ; $\mathrm{SO}_{4} \mathrm{Zn} .7 \mathrm{H}_{2} \mathrm{O}$; J.T. Baker, Mexico) and zinc gluconate stabilized with glycine (BZ; BioZn-AAS; Lipotech S.A., Argentina). Therefore we had the following groups: Group 1 receiving a diet with $2 \mathrm{ppm}$ of zinc as zinc sulfate, groups 2, 3 and 4 receiving diets with 9,20 and $34 \mathrm{ppm}$ of zinc as zinc sulfate; and groups 5, 6 and 7 receiving diets with 9, 20 and $34 \mathrm{ppm}$ of zinc as zinc gluconate stabilized with glycine. All zinc sources were provided in the yogurt vehicle added at $10 \%$ of the final diet. 
All diets and distilled water were provided ad libitum to the animals. Food intake was noted daily and animals were weighed three times a week. After 21 days of treatment with their respective diets, the animals were sacrificed and femur samples were extracted for further analysis.

\section{Bone and Diets Analysis}

The zinc content from experimental diets and femur bone samples was measured by atomic absorption spectrophotometry after a mineralization procedure previously described [10].

\section{Mathematical Models and Statistical Analysis of the Re- sults}

All data were analyzed using a computer statistical program (Graph Pad PRISM®, Version 3.00, 1999, GraphPad Software Inc., San Diego CA, USA). Body weight gain, femur weight and femur zinc content from all groups were compared by a two-way analysis of variance (ANOVA). We applied the simple effects test for the different levels of the two studied factors: doses and sources. A post-hoc test was carried out in order to find the differences between each dose, applying the Dunnett test. $\mathrm{p}<0.05$ was taken as the level of significance [11].

For the relative bioavailability analysis, body weight gain, femur weight and femur zinc contents were analyzed by means of mathematical models previously described elsewhere $[8,12]$. Briefly, group 1 with the diet containing 2 ppm of zinc was taken as a basal group for calculations, and then each source was separately analyzed taken into account the increasing in zinc dietary concentrations. Body weight gain and femur weight were analyzed by a nonlinear regression using equation (1):

$\mathrm{Y}=\mathrm{Y}_{\max }\left(1-\mathrm{e}^{-\mathrm{kX}}\right)$

where $\boldsymbol{Y}_{\max }$ represents the asymptotic response at high levels of dietary zinc, $\boldsymbol{k}$ is the constant of the rate of assimilation of the source in the matrix, and $\boldsymbol{X}$ is the zinc concentration. Alternatively to $\mathbf{k}$, we used $\mathbf{t}_{\mathbf{1} / \mathbf{2}}$ for bioavailability analysis, as the dietary zinc level needed to promote one half of the maximum response. This parameter can be obtained from equation, as $\mathbf{t}_{\mathbf{1} / \mathbf{2}}$ is $\ln 2 / \mathrm{k}$. Log values of femur zinc content were analyzed by linear regression taking into account results up to $20 \mathrm{ppm}$. Slopes and Y intercepts were determined using the log values of total femur zinc content. Therefore, all parameters obtained as a consequence of the analysis explained above were then compared in order to determine the relative bioavailability of zinc from the source under evaluation (BZ) with regard to the source taken as the reference standard (SZ).

\section{RESULTS}

Table 1 shows the results for body weight gain, femur weight and femur zinc content for the 7 groups. Group 1, fed with the basal diet ( $2 \mathrm{ppm}$ of $\mathrm{Zn}$ ), shows the lowest values for all parameters described and it is evident that an increase in the zinc diet content, using any of the sources (BZ or SZ) shows higher values for body weight gain, femur weight and femur zinc content $(\mathrm{p}<0.05)$.

For body weight gain, two dose- response curves were represented on Fig. (1), (one for each source) as a result from a nonlinear regression analysis. Parameters provided for the calculated curves and the relative zinc bioavailability (RBZ) [12], are shown in Table 2. It can be noted that the maximum body weight gain ( $\mathrm{Y}_{\max }$ for body weight gain) is higher for those animals fed with $\mathbf{B Z}$ (62.66 g) compared to those fed with SZ $(55.65 \mathrm{~g})$. However, the $\mathrm{t}_{1 / 2}$ to achieve that body weight is lower for SZ than for BZ. According to this analysis, the average RBZ of $\mathbf{B Z}$ calculated as taken into account the $\mathrm{Y}_{\max }$ and the $\mathrm{Y}_{\max } / \mathrm{t}_{1 / 2}$ of the two different sources is 0.95 .

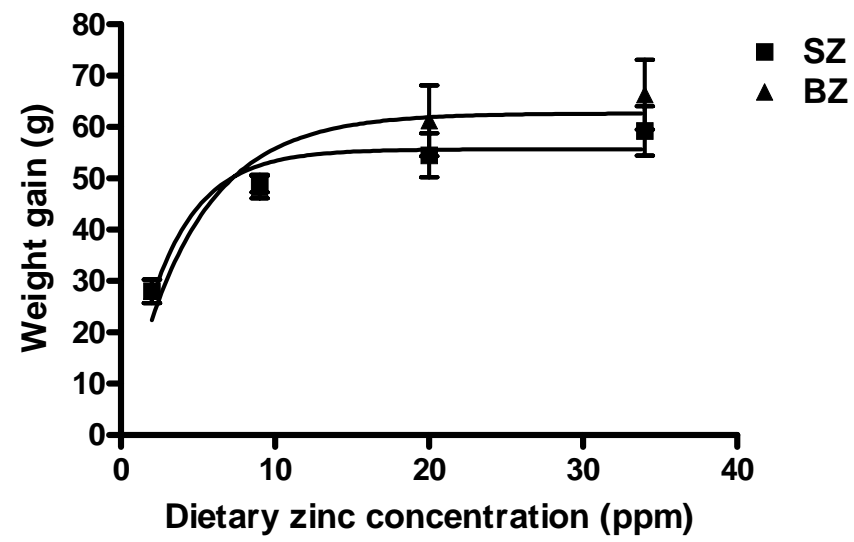

Fig. (1). Body weight gain versus dietary zinc content of SZ (ש) and $\mathrm{BZ}(\boldsymbol{\Delta})$. Values are expressed as means $\pm \mathrm{SD}$.

Table 1. Body Weight Gain, Femur Weight and Femur Zinc Content for the Different Diets Expressed as Mean \pm SD. BZ Stands for Zinc Gluconate Stabilized with Glycine and SZ for Zinc Sulfate. Results not Sharing a Superscript are Significantly Different, $\mathrm{p}<0,05$

\begin{tabular}{|c|c|c|c|c|c|}
\hline Group Under Study & Zinc Source & Zinc Diet Content (ppm) & Weight Gain (g) & Femur Weight (g) & Femur Zinc Content (ppm) \\
\hline 1 & Basal (SZ) & 2.0 & $28 \pm 2^{\mathrm{a}}$ & $0.26 \pm 0.05^{\mathrm{a}}$ & $16 \pm 3^{\mathrm{a}}$ \\
\hline 2 & \multirow{3}{*}{ SZ } & 9.0 & $49 \pm 2^{\mathrm{bc}}$ & $0.42 \pm 0.02^{\mathrm{b}}$ & $40 \pm 4^{\mathrm{b}}$ \\
\hline 3 & & 20.0 & $55 \pm 4^{\mathrm{c}}$ & $0.44 \pm 0.03^{\mathrm{b}}$ & $89 \pm 9^{c}$ \\
\hline 4 & & 34.0 & $59 \pm 5^{\mathrm{d}}$ & $0.43 \pm 0.05^{\mathrm{b}}$ & $100 \pm 9^{\mathrm{d}}$ \\
\hline 7 & $\mathrm{BZ}$ & 34.0 & $66 \pm 7^{d}$ & $0.6 \pm 0.1^{\mathrm{c}}$ & $110 \pm 14^{\mathrm{d}}$ \\
\hline
\end{tabular}


Table 2. Parameters of Curves Fitted to Body Weight Gain for Zinc Sources SZ and BZ: $\mathbf{R}^{2}, \mathbf{Y}_{\max }$ and $\mathbf{Y}_{\max } / \mathbf{t}_{1 / 2}$ and $\mathbf{R B Z} \mathrm{Ymax}_{\mathrm{max}}$ Determined by the Ratio of the $\mathbf{Y}_{\max }, \mathbf{R B Z} \mathbf{Y m a x}_{\mathbf{Y} / 2} t_{1 / 2}$ as Determined by Ratio of $\mathbf{Y}_{\max } / t_{1 / 2}$ and Average $\mathbf{R B Z}$

\begin{tabular}{|c|c|c|c|c|c|c|c|}
\hline Zinc Source & $\mathbf{R}^{2}$ & $\mathbf{Y}_{\max }(\mathrm{g})$ & $\mathbf{R B Z}_{\mathrm{Ymax}}$ & $\mathbf{t}_{1 / 2}(\mathbf{g})$ & $\mathbf{Y}_{\max } / \mathbf{t}_{1 / 2}$ & $\mathbf{R B Z}_{\mathrm{Ymax} /} t_{1 / 2}$ & RBZ \\
\hline SZ & 0.9489 & 55.65 & 1.00 & 2.17 & 25.64 & 1.00 & 1.00 \\
\hline
\end{tabular}

For femur weight dose-response curves, a similar analysis resulted as that for body weight gain and is represented in Fig. (2). From the non linear regression analysis, $\mathrm{Y}_{\max }$ and $\mathrm{t}_{1 / 2}$ were also calculated and are shown in Table 3. Average RBZ was 0.95 for $\mathbf{B Z}$ compared to $\mathbf{S Z}$.

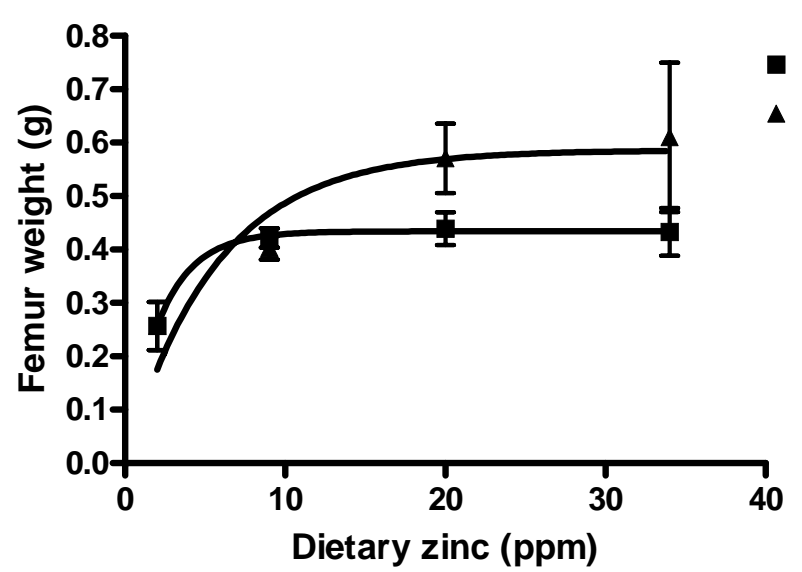

Fig. (2). Femur weight gain versus dietary zinc content of SZ ( and $\mathrm{BZ}(\boldsymbol{\Delta})$. Values are expressed as means $\pm \mathrm{SD}$.

The analysis for the femur zinc content appears in Fig. (3) where a log of femur zinc content versus dietary zinc content curve resulted from a linear regression adjustment. Thus, the analyzed parameter was the slope for each curve although the $\mathrm{Y}_{\text {intercept }}$ and the $\mathrm{R}^{2}$ of each curve are also shown in Table 4. In the case of $\mathbf{S Z}$ the slope was $0.038 \pm 0.007$ and for BZ was $0.054 \pm 0.008$. Finally, the RBZ was obtained by calculating the ratio of the slopes. According to this result, the RBZ for $\mathbf{B Z}$ is 1.10 with regard to $\mathbf{S Z}$.

\section{DISCUSSION}

Methods for assessing zinc bioavailability include labelling techniques and the comparison of the nutritional responses derived from dose response curve fitting. Indeed, the combination of mathematical and biological analysis will provide the best information to compare zinc bioavailability of different sources. In our study, we considered a model of non linear regression to analyze body weight gain and femur weight and of linear regression to analyze the femur zinc content. These analyses yielded a series of parameters that could be compared in order to determine the RBZ of a zinc source like zinc gluconate stabilized with glycine provided as a fortificant in yogurt vehicle, using zinc sulfate added in the same vehicle as the reference standard.

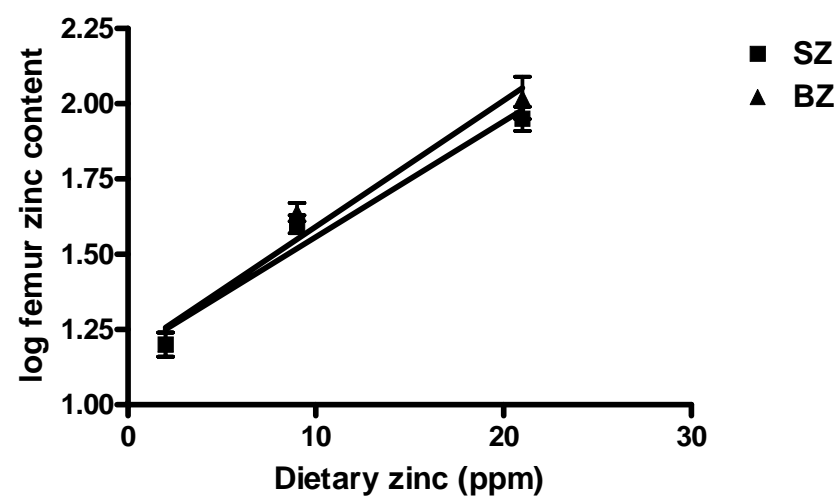

Fig. (3). Log femur zinc content versus dietary zinc content of SZ $(\boldsymbol{\square})$ and BZ $(\boldsymbol{\Delta})$. Values are expressed as means \pm SD.

$\mathrm{Y}_{\max }$ is an indicator of the maximum effect that can be achieved when the zinc content in the diet is modified. This value can be of much use to evaluate the limitation of an analyzed source for possible food fortification in a given nutritional matrix. In this sense, the biological efficiency of the added source may be evaluated by means of the rate of maximum effect it can be achieved according to its content in diet. $\mathrm{Y}_{\max } / \mathrm{t}_{1 / 2}$ also indicates the nutrient efficiency, and a high value for this parameter is desirable in order to consider the quantities of the nutrient source that will be added to the diet to achieve a certain level of the maximum effect. Therefore, since both $Y_{\max }$ and $Y_{\max } / t_{1 / 2}$ matter when evaluating a nutritional effect for a given zinc source, the RBZ can be calculated by the comparison of these parameters. On the other hand, the slope in the femur zinc content analysis may be interpreted in an analogous way than $Y_{\max }$ and $\mathrm{Y}_{\max } / \mathrm{t}_{1 / 2}$ with regard to the nutritional efficiency of the studied source. In this study, the different biological parameters analyzed to compare the bioavailability of zinc sulfate and zinc gluconate stabilized with glycine in yogurt, such as body weight gain, femur weight and femur zinc content, which are directly related to zinc metabolism and are represented by

Table 3. Parameters of Curves Fitted to Femur Weight for Zinc Sources $S Z$ and $B Z: R^{2}, Y_{\max }$ and $Y_{\max } / t_{1 / 2}$ and $R B Z Z_{Y_{m a x}}$ as Determined by the Ratio of the $Y_{\max }, \mathbf{R B Z} \mathrm{Ymax}_{\mathbf{t} 1 / 2}$ as Determined by ratio of $\mathrm{Y}_{\max } / \mathrm{t}_{1 / 2}$ and Average $\mathrm{RBZ}$

\begin{tabular}{|c|c|c|c|c|c|c|c|}
\hline Zinc Source & $\mathbf{R}^{2}$ & $\mathbf{Y}_{\max }(\mathbf{g})$ & $\mathbf{R B Z}_{\mathbf{Y m a x}}$ & $\mathbf{t}_{\mathbf{1} / 2}(\mathbf{g})$ & $\mathbf{Y}_{\max } / \mathbf{t}_{\mathbf{1} / \mathbf{2}}$ & $\mathbf{R B Z}_{\mathbf{Y m a x} / \mathbf{1} / \mathbf{2}}$ & $\mathbf{R B Z}$ \\
\hline \hline SZ & 0.9981 & 0.4342 & 1.00 & 1.55 & 0.280 & 1.00 & 1.00 \\
\hline BZ & 0.8549 & 0.5859 & 1.35 & 3.90 & 0.150 & 0.54 \\
\hline
\end{tabular}


Table 4. Parameters of Linear Regression of Femur Zinc Content for Zinc Sources SZ and BZ: $\mathbf{Y}_{\text {intercept }}, \mathrm{Slopes} \mathbf{R}^{2}$ and RBZ as Determined by the Slope Ratio

\begin{tabular}{|c|c|c|c|c|}
\hline Zinc Source & $\mathbf{R}^{2}$ & $\mathbf{Y}_{\text {intercept }}(\mathbf{p p m})$ & Slope & $\mathbf{R B Z}$ \\
\hline \hline SZ & 0.9646 & 1.17 & 0.038 & 1.00 \\
\hline BZ & 0.9632 & 1.17 & 0.042 & 1.10 \\
\hline
\end{tabular}

$\mathrm{Y}_{\max }, \mathrm{Y}_{\max } / \mathrm{t}_{1 / 2}$ and the slope of the curves as mathematical parameters, rendered similar results in average.

Since not only the bioavailability is important when analyzing a nutrient source and other properties should be taken into consideration, zinc gluconate stabilized with glycine should also be evaluated with regard to the organoleptic properties of the food it will be fortifying. Although this was not the aim of our study, some preliminary tests showed that this source is suitable for yogurt and infant dessert fortification since it causes none of sensorial modifications of these food vehicles in contrast with zinc sulfate.

\section{CONCLUSION}

For all the above mentioned, zinc gluconate stabilized with glycine may be considered as a suitable source of zinc for food fortification in a yogurt matrix.

\section{REFERENCES}

[1] Swenerton H, Hurley V. Severe zinc deficiency in male and female rats. J Nutr 1968; 95: 8-18 .

[2] Brandão-Neto V, Stefan V, Mendoça BB, Bloise W, Castro A V. The essential role of zinc in growth. Nutr Res 1995; 15: 335-358.
[3] Prasad S. Zinc in human health: effect of zinc on immune cells. Mol Med 2008; 14: 353-357.

[4] Prasad S, Overleas D, Rajasekaran G. Essential micronutrient elements. Am J Clin Nutr 1970; 23: 581-591.

[5] Prasad AS. Discovery and importance of zinc in human nutrition. Fed Proc 1984; 43: 2829-2834.

[6] O’Dell B. History and status of zinc in nutrition. Fed Proc 1984; 43: 2821-2822.

[7] Salgueiro J, Leonardi N, Zubillaga M, et al. Normal growth rate in rats is recovered after a period of zinc deficiency by restoration of zinc supply by means of zinc- fortified petit Suisse cheese. Biol Trace Element Res 2005; 104: 269-273.

[8] Salgueiro J, Leonardi N, Zubillaga M, et al. Determination of relative bioavailability of zinc in a petit suisse cheese using weight gain and bone zinc content in rats as markers. Biol Trace Element Res 2005; 104: 261-267.

[9] Reeves PG, Nielsen FH, Fahey Jr. GC. AIG-93 Purified diets for laboratory rodents: final report of the American Institute of Nutrition Ad Hoc Writing Committee on the Reformulation of the AIG76A Rodent Diet. J Nutr 1993; 123: 1939-1951.

[10] Lysionek AE, Zubillaga MB, Calmanovici GP, et al. Modification of the ferrozine technique to analyze iron contents in different foods. Biol Trace Element Res 1998; 65: 87- 94.

[11] Sokal RR, Rohlf F J, Freeman WH. Biometry. San Francisco 1996.

[12] Mercer LP, Flodin NW, Morgan PH. New methods for comparing the biological efficiency of alternate nutrient sources. J Nutr 1978; 108: $1244-1249$. 